\title{
Teilhabeziel Arbeit
}

\section{Modularisierte Angebote für Menschen mit Behinderung}

\author{
Claudia Zinke
}

\begin{abstract}
Die sich seit vier Jahren hinziehende Diskussion über eine Reform der Eingliederungshilfe betrifft auch die Institution der Werkstatt für behinderte Menschen. Sollte es zu einer formalisierten "Modularisierung " der Angebote für die Teilhabe an Arbeit kommen, steht zudem die Ganzheitlichkeit der Eingliederungshilfe auf dem Spiel.
\end{abstract}

Der nachfolgende Beitrag befasst sich mit der Teilhabe am Arbeitsleben aus dem Blickwinkel der Werkstatt für Menschen mit Behinderung. In den Ausführungen wird eine erste Bewertung von Vorschlägen zur Weiterentwicklung der Eingliederungshilfe für Menschen mit Behinderung vorgenommen, und zwar bezogen auf das Ziel, künftig die Leistungen zur Teilhabe am Arbeitsleben personenzentriert und gleichzeitig modularisiert zu erbringen. Aufgegriffen werden dabei die Fragestellungen: Was bedeutet Arbeit und Teilhabe am Arbeitsleben für Menschen mit Behinderung? Und sollte es für die betroffenen Menschen besser ein umfassendes oder ein in Einzelteilen zergliedertes modularisiertes - Angebot geben?

Die Werkstatt für behinderte Menschen ist eine Einrichtung zur Teilhabe am Arbeitsleben. Dieser Institution ist im Sozialgesetzbuch IX (Rehabilitation und Teilhabe) ein eigenes Kapitel gewidmet,

\footnotetext{
Claudia Zinke ist Referentin für Gesundheitshilfe, Behindertenhilfe und Psychiatrie beim Paritätischen Gesamtverband in Berlin. Der vorliegende Beitrag entstand auf der Grundlage eines gemeinsamen Referats mit Dr. jur. Alexander Vater, ehemaliger stellvertretender Geschäftsführer der Johannes Diakonie Mosbach und Mitglied im Vorstand des Bundesverbandes evangelische Behindertenhilfe.
}

E-Mail behindertenhilfe@paritaet.org in dem Bedingungen und Voraussetzungen geregelt werden. Hinzu kommt die Werkstättenverordnung, in der neben der Struktur der Werkstatt (Eingangsverfahren, Berufsbildungsbereich, Arbeitsbereich) weitere differenzierte Regelungen (Größe, Beschäftigungszeit etc.) getroffen wurden, die kaum Spielraum lassen.

Im gesamten Sozialrecht gibt es wohl kaum eine Institution, die so intensiv kodifiziert ist, wie die Werkstatt für behinderte Menschen. Die Wurzeln der heutigen Werkstätten gehen bis in die 1950er Jahre zurück. Bei den damaligen Bemühungen der Wohnstätten, ein Beschäftigungsangebot für die Menschen zu schaffen, stand weder der Gesichtspunkt der Produktion oder Rentabilität oder die Arbeitsentlohnung im Vordergrund. Es sollten für die Menschen mit Behinderung befriedigende Betätigungsmöglichkeiten geschaffen werden, die darauf ausgerichtet waren, möglichst auch die Schwächsten einzubeziehen und seien es nur wenige Stunden am Tage.

Mit Unterstützung der Elternvereinigung der Lebenshilfe entstanden die »beschützenden Werkstätten «, die sich von Anfang an als » Glied der Wirtschaft « verstanden und dem Faktum Produktivität mehr Bedeutung zukommen ließen. Begründet wurde dies u. a. damit, dass die Gesellschaft es sich nicht erlauben könne, die Kräfte der großen Gruppe der Menschen mit geistiger Behinderung ungenutzt zu lassen.

Erst in den 1960er Jahren wurde - ausgehend von einem internationalen Symposium - die soziale Rehabilitation als gleichwertiges Ziel neben die arbeitsmäßige Rehabilitation gestellt. Von da an wurde die Teilhabe für Menschen mit einer geistigen Behinderung an einer geschützten Beschäftigung nicht als Sache der Wohltätigkeit, sondern als Rechtsanspruch verstanden. Kostenträger war allein die Sozialhilfe. Erst 1969 kam mit dem Arbeitsförderungsgesetz die Bundesanstalt für Arbeit als Träger insbesonde- re für berufsfördernde Leistungen für Menschen mit Behinderung hinzu. Infolge dessen wurde die bisher auf soziale Eingliederung ausgerichtete Konzeption auf eine arbeitsmarkt-, produktions- und leistungsorientierte Konzeption erweitert. In dieser Zeit wurde das bis heute geltende konzeptionelle Fundament für die Werkstätten mit vier wesentlichen Merkmalen gelegt:

- Die Doppelorientierung der Werkstatt: Auf der einen Seite soll die Werkstatt Dauerarbeitsplätze für behinderte Menschen zur Verfügung stellen, die wegen Art oder Schwere ihrer Behinderung auf dem allgemeinen Arbeitsmarkt nicht vermittelt werden können. Auf der anderen Seite hat die Werkstatt die Verpflichtung, Beschäftigte auf den allgemeinen Arbeitsmarkt vorzubereiten und zu vermitteln.

- Das unterschiedliche Leistungsrecht: Für die Leistungen in der Werkstatt sind die Bundesanstalt für Arbeit und der Träger der Sozialhilfe mit jeweils unterschiedlichen Leistungsvoraussetzungen und Zielen zuständig.

- Die Einführung von Aufnahmekriterien: Voraussetzung für Aufnahme von Menschen mit Behinderung ist, dass diese ein Mindestmaß wirtschaftlich verwertbarer Arbeitsleistung erbringen.

- Die Bindung von Nachteilsausgleichen an die Institution: Menschen mit Behinderung, die in anerkannten Werkstätten beschäftigt sind, werden in der gesetzlichen Krankenversicherung, der sozialen Pflegeversicherung, der gesetzlichen Rentenversicherung und der gesetzlichen Unfallversicherung versichert.

Bis vor wenigen Jahren waren Werkstätten für behinderte Menschen unbestritten das dominierende Beschäftigungsangebot. Es war selbstverständlich, dass Menschen mit einer geistigen Behinderung nach Abschluss der Schule berufliche Bildungsmaßnahmen in den Werkstätten wahrnahmen und im Anschluss 
daran in der Regel lebenslang den Arbeitsbereich besuchten.

Seit einigen Jahren wird die Institution Werkstatt jedoch immer wieder hinterfragt. Ursache für die aufkommende Kritik ist vor allem die zunehmende Anzahl von Beschäftigten und die damit verbundenen steigenden Kosten. Die Bundesregierung hat $u$. a. diese Kritik aufgegriffen und sich im Jahre 2005 im damaligen Koalitionsvertrag von CDU/CSU und SPD darauf verständigt, dass mehr Menschen mit Behinderung die Möglichkeit haben, außerhalb von Werkstätten für behinderte Menschen ihren Lebensunterhalt im allgemeinen Arbeitsmarkt erarbeiten zu können. Um dieses Ziel zu erreichen, wurden in den letzten Jahren neue Maßnahmen gesetzlich verankert. Dazu gehören das Instrument zur Diagnose der Arbeitsmarktfähigkeit besonders betroffener Menschen (DIA-AM, 2008) und das Gesetz zur Einführung »Unterstützter Beschäftigung «(UB, 2009).

Die Arbeits- und Sozialministerkonferenz hat sich seit 2007 in ihren Beschlüssen zur Weiterentwicklung der Eingliederungshilfe jährlich mit der Teilhabe am Arbeitsleben befasst. Als Ergebnis liegen nach vier Jahren intensiver Diskussion Eckpunkte vor, auf deren Grundlage noch in diesem Jahr ein Arbeitsentwurf für ein Gesetz zur Weiterentwicklung der Eingliederungshilfe erstellt werden soll.

Grundsätzliches Ziel dieser Reform soll sein, das Leistungsspektrum von einer bisher einrichtungsorientierten $\mathrm{zu}$ einer personenzentrierten Leistung auszurichten. Für die Teilhabe am Arbeitsleben sind u. a. die Zulassung "alternativer « Anbieter in Ergänzung zur Werkstatt, die Einführung eines Lohnkostenzuschusses in "geeigneten Fällen «, die Einführung eines beruflichen Orientierungsverfahrens, die Weiterentwicklung der Fachausschüsse und die Einführung von Leistungsmodulen von Bedeutung. An der Zielgruppe und den bisherigen Zugangsvoraussetzungen für eine Werkstatt für Menschen mit Behinderung wird festgehalten. Grundsätzlich könnten solche Entwicklungen den Übergang auf den allgemeinen Arbeitsmarkt oder die Inanspruchnahme Persönlicher Budgets für die Teilhabe am Arbeitsleben fördern, zumal über Regelungen für ein Rückkehrrecht in die Werkstatt nachgedacht wird.

Die seit Jahren intensiv bestehende Diskussion zur Reform der Eingliede- rungshilfe und den Gedanken der personenorientierten der modularisierten Leistungen haben verschieden Akteure aufgegriffen. Dazu gehören beispielsweise das Projekt »JobBudget « im Rahmen des Persönlichen Budgets und die Überlegungen der Bundesarbeitsgemeinschaft überörtlicher Sozialhilfeträger in ihrer Stellungnahme zur Weiterentwicklung der Eingliederungshilfe. Wesentlicher Kern der Vorschläge der überörtlichen Sozialhilfeträger ist, dass die bisher in den $\mathbb{S} \mathbb{S} 39$ bis 41 SGB IX beschriebenen Werkstattleistungen gesetzlich modularisiert werden sollen. Hierfür werden sechs Module vorgeschlagen, die unterscheiden zwischen Leistungen für berufliche Bildung, Beschäftigung zu einem angemessenen Arbeitsentgelt, Arbeitsbegleitung, Weiterentwicklung der Persönlichkeit, Förderung des Übergangs auf den allgemeinen Arbeitsmarkt und sonstigen Leistungen (z.B. Beförderung). Darüber hinaus soll der Sozialhilfeträger allein in einem Hilfeplanverfahren feststellen, welche Leistungen (Module) der einzelne behinderte Mensch benötigt (Hilfebedarf).

Die Leistungs-Module können unverändert auf der Grundlage der bestehenden gesetzlichen Bestimmungen in einer Werkstatt für behinderte Menschen erbracht werden. Alternativ dazu sollen andere Leistungserbringer die Leistungen auch einzeln anbieten können. Allerdings soll im Gegensatz zur Werkstatt bei anderen Leistungserbringern eine förmliche Anerkennung nicht erforderlich sein. Die Bundesarbeitsgemeinschaft überörtlicher Sozialhilfeträger ist weiterhin der Auffassung, die geplante Personenzentrierung und die Modularisierung der Leistungen zur Teilhabe am Arbeitsleben böte die Chance für ein neues Vergütungssystem. Dieses neue Vergütungssystem soll sich nicht mehr nur an den Kosten der Werkstatt orientieren, sondern auch - auf Dauer überwiegend oder ausschließlich - am Bedarf des Leistungsempfängers. Die einzelnen Module könnten im Grundsatz trägerunabhängig auf der Länderebene kalkuliert werden. Weitere Vorschläge betreffen die Schaffung von Vergütungsgruppen, einheitliche prozentuale Zuschläge für Sachkosten, den Investitionsbetrag und die Zuordnung des Mittagessens zum Lebensunterhalt.

\section{Berufliche Bildung, Beschäftigung und Persönlichkeitsentwicklung kennzeichnen die Werkstatt}

Nach \ 136 Abs. 1 SGB IX hat die Werkstatt den leistungsberechtigten behinderten Menschen eine angemessene berufliche Bildung und eine Beschäftigung zu einem ihrer Leistung angemessenen Arbeitsentgelt aus dem Arbeitsergebnis anzubieten. Darüber hinaus hat sie es zu ermöglichen, die Persönlichkeit weiterzuentwickeln.

Berufliche Bildung, Beschäftigung und Persönlichkeitsentwicklung sind also die Trias, die Werkstattarbeit kennzeichnen. Insofern unterscheidet sich die Beschäftigung in einer Werkstatt für behinderte Menschen vom allgemeinen Arbeitsmarkt, der durch den Austausch von Leistung gegen Entgelt geprägt ist. Eine auf Dauer angelegte berufliche Bildung und Maßnahmen zur Weiterentwicklung der Persönlichkeit gehören schwerpunktmäßig nicht zu den Zielen der Unternehmen auf dem allgemeinen Arbeitsmarkt.

Damit dem unterschiedlichen Leistungsvermögen der Menschen mit Behinderungen Rechnung getragen werden kann, hat der Gesetzgeber eine Vielfalt bei einem Angebot an Arbeitslätzen in Werkstätten gewollt und dies im $\mathbb{} 136$ Abs. 1 SGB IX zum Ausdruck gebracht. Die Werkstatt soll demnach über ein möglichst breites Angebot an Berufsbildungs- und Arbeitsplätzen verfügen. Es ist sicherlich nichts dagegen einzuwenden, dass berufliche Bildung, Beschäftigung und Persönlichkeitsentwicklung, die im bisherigen Verständnis der Institutionsbezogenheit pauschal angeboten wurden, künftig differenzierter beschrieben und als Module bezeichnet werden. Es ist auch nicht zu beanstanden, dass anstelle der heute im SGB IX beschriebenen Leistungen, " personenorientierte Leistungen « definiert werden sollen, die der Mensch mit Behinderung unabhängig von Ort und Träger der Leistungserbringung - auch in Form eines Persönlichen Budgets - in Anspruch nehmen kann. Voraussetzung wäre allerdings, dass die erwähnte Trias - berufliche Bildung, Beschäftigung und Persönlichkeitsentwicklung - erhalten bleiben. Ob dies Module oder Leistungskomplexe künftig sein können, ist von verschiedenen Bedingungen abhängig. Die Vorstellungen der Bundesarbeitsgemeinschaft überörtlicher 
Sozialhilfeträger sind bezogen auf die Trennung zwischen Leistungen zur Weiterentwicklung der Persönlichkeit und denen der Beschäftigung, das alleinige Feststellen der Sozialhilfeträger der erforderlichen Module und die Unterscheidung zwischen »erforderlichen " und »übrigen Leistungen « bei weiterem Bedarf kritisch zu betrachten und werfen mindestens dreizehn Fragen auf:

Erste Frage: Wird es Pflicht und Zusatzleistungen geben? Oder wird geplant, Leistungen, die bisher der Teilhabe am Arbeitsleben zugeordnet waren, künftig anderen Maßnahmen anzugliedern, beispielsweise der Pflege oder der Teilhabe am Leben in der Gemeinschaft mit veränderten Bedingungen bei der Einkommensund Vermögensprüfung und bei der Unterhaltsheranziehung der Angehörigen?

Zweite Frage: Lassen sich die künftigen Module von denen der »Unterstützten Beschäftigung « und der »Diagnose der Arbeitsmarktfähigkeit « abgrenzen?

Dritte Frage: Werden die Menschen künftig noch mehr zwischen den Leistungsträger hin- und herverwiesen und erleben wir möglicherweise eine Renaissance der Auseinandersetzungen der 1960er Jahre zwischen den Vorstellungen der Agentur für Arbeit und der Sozialhilfe?

Vierte Frage: Welche Funktion wird künftig der Fachausschuss haben? Soll die multiprofessionelle und interdisziplinäre Feststellung der Werksattfähigkeit zugunsten einer alleinigen Feststellung durch den Sozialhilfeträger aufgegeben werden?

Fünfte Fragen: Wer übernimmt die Verantwortung und wer organisiert die Kooperation im Prozess der Umsetzung, bezogen auf die Qualität der Leistungen, wenn die Module von unterschiedlichen Anbietern erbracht werden?

Sechste Frage: Wer wird die Menschen zu diesen differenzierten Leistungen beraten und wer übernimmt die Beratungsleistungen?

Siebte Frage: Wird es Verpflichtungen für die Leistungsanbieter geben, Kooperationsvereinbarungen einzugehen?

Achte Frage: Wird es Veränderungen geben bezogen auf den Status (arbeitnehmerähnliches Rechtsverhältnis) für den Menschen mit Behinderung geben, wenn er die Module von unterschiedlichen Anbietern erhält?

Neunte Frage: Mit wem schließt der behinderte Mensch den Vertrag für die Leistungen der Module, wenn dies unter- schiedliche Anbieter sind? Wird er mehrere Verträge schließen müssen?

Zehnte Frage: Von wem erhält er sein Arbeitsentgelt?

Elfte Frage: Wo wird der behinderte Mensch künftig seine Mitwirkungsrechte geltend machen, welchem »Werkstattrat « schließt er sich an?

Zwölfte Frage: Nach den Vorstellungen der Bundesregierung können die alternativen Anbieter für Leistungen auch die Integrationsfachdienste sein - kann dieses neue Angebot kompatibel mit den bestehenden Aufgaben der Integrationsfachdiensten im Rahmen der Ausgleichsabgabe sein? Und: Werden die Leistungen der beruflichen Bildung künftig den Vorgaben der öffentlichen Ausschreibung unterliegen?

Dreizehnte Frage: Werden die von der Bundesregierung gewollten künftigen »alternativen Anbieter « die gleiche Qualität und den selben Umfang der Leistung wie die Werkstätten erbringen müssen, beispielsweise die Sicherstellung der Fahrdienstleistungen zum Arbeitsplatz oder wird es eine "Werkstatt light « geben?

Nach jetzigen Einschätzungen besteht die Gefahr, dass die Ganzheitlichkeit der Eingliederungshilfe mit der Einführung von Modulen aufgegeben wird. Beispielhaft sei hier die Leistung für die Einnahme des Mittagessens in der Werkstatt benannt. Leistungsmodule oder Komplexleistungen machen nur Sinn, wenn klar ist, dass auch ein Leistungsträger dafür geradesteht. Schon heute gibt es genügend rechtliche Auseinandersetzungen aufgrund des gegliederten Systems.

Für Menschen mit Behinderung kann eine Alternative zur Teilhabe am Arbeitsleben in der Werkstatt nur eine Beschäftigung unter vergleichbaren Kriterien sein. Dazu gehört, dass persönlichkeitsbildende Maßnahmen in der beruflichen Teilhabe nicht zur Disposition stehen. Eine Überprüfung, ob nicht Teile der persönlichkeitsbildenden Leistungen der Teilhabe am Leben in der Gemeinschaft zugeordnet werden können, darf nicht vorgenommen werden. Fähigkeiten der Persönlichkeit,beispielsweise der Kommunikation, sind Fähigkeiten, die für eine Teilhabe sowohl am Arbeitsleben als auch in der Freizeit und im Wohnen eine wichtige Voraussetzung darstellen, sie sind deshalb auch jeweils in diesen Bereichen entsprechend zu fördern oder durch Assistenz zu unterstützen.

Weiterhin besteht die Gefahr, dass bei einer Modularisierung der Leistungen ein abgeschlossener Leistungskatalog den Individualitäten der Menschen kaum gerecht werden kann und möglicherweise nur einen Teil der Leistungen decken wird. Dies stellt eine besondere Gefahr für Menschen mit hohem Unterstützungsbedarf dar. Beispielhaft sei hier die Pflegeversicherung genannt, deren Umsetzung durch Leistungskomplexe geprägt ist.

Allerdings können Module die Chance für einen bundesweit einheitlichen Leistungskatalog sein. Personenorientierte Leistungen brauchen individuelle Lösungen. Abschließende Module können dem nicht gerecht werden. Personenorientierte Leistungen brauchen eine Änderung des restriktiv geregelten Werkstättenrecht. Vorstellbar wäre ein Verordnung vom Bundesgesetzgeber, in der die Qualität der Teilhabeleistungen gleichermaßen für Werkstätten und für »alternative Anbieter « geregelt werden könnte.

Personenorientierte Leistungen schließen auch Menschen mit hohem Unterstützungsbedarf ein. Insofern wäre der Begriff »Mindestmaß wirtschaftlich verwertbarer Arbeitsleistung « in $\$ 136$ SGB IX zu streichen, so dass für alle Menschen mit Behinderung der Zugang zu einer für sie angemessenen Beschäftigung möglich wird.

Personenorientierte Leistungen brauchen Vertrauen der Leistungsträger in die Leistungsanbieter. Misstrauen befördert verstärktes Kontrollabläufe, die zu kleinteiligen Maßnahmen und Vergütungen führen, und kaum Spielräume für individuelle Lösungen zu lassen.

\section{Resümee}

Differenzierte Leistungen können mehr Wahlmöglichkeiten für solche Menschen mit Behinderungen schaffen, die einen Rechtsanspruch auf Beschäftigung in einer Werkstatt haben. Allerdings kommen Zweifel auf, ob die Vorstellungen der überörtlichen Sozialhilfeträger den Beschlüssen der Arbeits- und Sozialministerkonferenz und den wiederholten Bekräftigungen der Bund-Länder-Arbeitsgruppe übereinstimmen. Die Vertreter der beiden Gremien haben stets das Grundanliegen betont, nämlich Teilhabemöglichkeiten und Leistungen der Eingliederungshilfe für Menschen mit Behinderungen und deren Angehörigen nicht einzuschränken, zu ihrem Nachteil zu kürzen oder wegfallen zu lassen.

Eine schwierige, aber lohnenswerte Aufgabe für alle Beteiligten. 